\title{
O Estado da Arte a Respeito dos Estudos de Educação Ambiental Realizados em Comunidades Quilombolas no Brasil
}

\author{
The State of the Art Regarding Environmental Education Studies Carried out in \\ Quilombola Communities in Brazil
}

\author{
El Estado del Arte en Estudios de Educación Ambiental Realizados en \\ Comunidades Quilombolas en Brasil
}

\author{
Maurivan Vaz Ribeiro ${ }^{1}$ \\ Vanessa Maluf de Novais ${ }^{2}$ \\ Leila Reis Bastos ${ }^{3}$ \\ Rafaella Ferreira Rodrigues Almeida ${ }^{4}$ \\ Waléria Patrícia Brito de Oliveira ${ }^{5}$ \\ Katia Kopp ${ }^{6}$
}

\begin{abstract}
Resumo
O Brasil apresenta uma imensa diversidade étnica, linguística e cultural e, apesar do grande número de comunidades quilombolas no Brasil, estas ainda sofrem o descaso e a falta de reconhecimento de suas terras, aumentando, ainda mais, seus problemas sociais, como o acesso à saúde e ao saneamento básico. Do ponto de vista ambiental, os territórios das comunidades tradicionais, em geral, são bem preservados devido à grande relação dessas comunidades com a natureza, onde cada espécie de planta, grupo de animais, tipo de solo e paisagem possuem um uso prático ou religioso. Ações de educação ambiental têm se tornado uma ferramenta importante na busca pela identificação cultural e preservação da natureza das comunidades quilombolas. Apesar disso, poucos estudos tem sido publicados a respeito dessas ações, o que dificulta a identificação de lacunas de conhecimento em relação às comunidades quilombolas menos assistidas. $O$ presente trabalho buscou identificar, quantitativamente, os estudos de educação ambiental realizados em comunidades quilombolas. Foram realizadas buscas na plataforma Google Scholar por meio das seguintes palavras-chave: "Quilombolas", "Educação Ambiental" e "Comunidades tradicionais". Foram encontrados 65 estudos, a maior parte do tipo artigo científico. A Comunidade Quilombola Mata-Cavalo, localizada às margens da BR-MT 060, no estado do Mato Grosso-MT, foi a mais estudada, e o ano com maior número de publicações foi 2018. Os temas dos estudos encontrados variaram bastante, demonstrando o caráter multidisciplinar da educação ambiental. A maioria desses estudos foi realizada por universidades públicas, mostrando a importância da valorização dessas instituições.
\end{abstract}

Palavras-chave: Quilombolas. Educação ambiental. Comunidades tradicionais.

\section{Abstract}

\footnotetext{
${ }^{1}$ Mestre em Biodiversidade Animal pela Universidade Federal de Goiás - UFG, Doutorando em Ecologia e Conservação da Biodiversidade pela Universidade Estadual de Santa Cruz em Ilhéus-BA. E- mail: maurivan.bio@gmail.com

${ }^{2}$ Engenheira Ambiental pela Faculdade Araguaia. Email: nessamnovais@ gmail.com

${ }^{3}$ Engenheira Ambiental pelo Instituto Federal de Educação, Ciência e Tecnologia de Goiás - IFG. Email: leilarbastos@gmail.com

${ }^{4}$ Engenheira Ambiental pelo Instituto Federal de Educação, Ciência e Tecnologia de Goiás - IFG. Email: rafaella.redes@hotmail.com

${ }^{5}$ Engenheira Ambiental pela Faculdade Araguaia. Email: waleriapoliveira@yahoo.com.br

${ }^{6}$ Curso de Engenharia Ambiental e Sanitária, Escola de Engenharia Civil e Ambiental da Universidade Federal de Goiás - UFG. Email: kakopp@ufg.br
} 
Brazil presents an immense ethnic, linguistic and cultural diversity and, despite the large number of quilombola communities in Brazil, these still suffer from neglect and lack of recognition of their lands, further increasing their social problems, such as access to health and to basic sanitation. From an environmental point of view, the territories of traditional communities, in general, are well preserved due to the great relationship of these communities with nature, where each species of plant, group of animals, type of soil and landscape has a practical or religious use. Environmental education actions have become an important tool in the search for cultural identification and preservation of nature in quilombola communities. Despite this, few studies have yet been published about these actions, what makes the identification of knowlege gaps difficult in relation to the less assisted quilombola communities. The present work sought to quantitatively identify the environmental education studies carried out in quilombola communities. Searches were performed on the Google Scholar platform through the following keywords: "Quilombolas", "Environmental Education" and "Traditional Communities". Sixty-five studies were found, most of them scientific articles. The Mata-Cavalo Quilombola Community, located on the banks of BR-MT 06, in the state of Mato Grosso-MT, was the most studied community, and the year with the highest number of publications was 2018. The themes of the studies found were quite variable, demonstrating the multidisciplinary character of environmental education. Most of these studies were carried out by public universities, showing the importance of valuing these institutions.

Keywords: Quilombolas. Environmental education. Traditional communities.

\section{Resumen}

Brasil tiene una inmensa diversidad étnica, lingüística y cultural y, apesar de la gran cantidad de comunidades quilombolas en Brasil, estas aún sufren de abandono y falta de reconocimiento de sus tierras, lo que aumenta, aún más, los problemas sociales que enfrentan estas comunidades, como el acceso a la salud y al saneamiento básico. Desde el punto de vista ambiental, los territorios de las comunidades tradicionales, en general, se encuentran bien conservados debido a la gran relación de estas comunidades com la naturaleza, donde cada especie de planta, grupo de animales, tipo de suelo y paisaje tienen un carácter práctico o religioso. Las acciones de educación ambiental se han convertido en una herramienta importante em la busqueda de la identificación cultural y preservación de la naturaleza de las comunidades quilombolas. Apesar de esto, aún se han publicado pocos estudios sobre estas acciones, lo que dificulta la identificación de brechas de conocimiento en relación a las comunidades quilombolas menos asistidas. El presente trabajo buscó identificar, cuantitativamente, los estudios de educación ambiental realizados en comunidades quilombolas. Las busquedas se realizaron en la plataforma Google Scholar utilizando las siguientes palabras clave: "Quilombolas", "Educación ambiental" y "Comunidades tradicionales". Se encontraron sesenta y cinco estudios, la mayoría de ellos artículos científicos. La comunidad Quilombola MataCavalo ubicada a lo largo de la BR-MT 060 en el Estado de Mato Grosso-MT, fue la más estudiada y el año com mayor número de publicaciones fue el 2018. Los temas de los estudios encontrados fueron bastante variables, demostrando el carácter multidisciplinario de la educación ambiental, y llevados a cabo, em su mayoría, por universidades públicas, demostrando la importancia de valorar estas instituciones.

Palabras clave: Quilombola. Educación ambiental. Comunidades tradicionales.

\section{Introdução}

O Brasil apresenta uma imensa diversidade étnica, linguística e cultural (DIEGUES et al., 2000). Apenas com relação aos quilombolas, que estão distribuídos por, pelo menos, 24 estados brasileiros, 3.432 comunidades foram reconhecidas pela Fundação Palmares, órgão do governo federal brasileiro que trata dessas questões (FUNDAÇÃO PALMARES, 2020).

As comunidades quilombolas são formadas, atualmente, por descendentes de pessoas escravizadas que conseguiram escapar de prisões e senzalas no Brasil, constituindo grupos de resistência. Estes viviam livres, de acordo com a sua cultura originária, de matriz africana 
(SOUZA et al., 2009; PALMARES, 2020). Tais comunidades, apesar de terem, no art. 68 da Constituição Federal, o reconhecimento de suas terras como propriedade definitiva, muitas ainda vivenciam conflitos com grileiros de terras, pois, na prática, esse reconhecimento não se efetiva (AMORIM; GERMANI, 2005; LEITE, 2010; OLIVEIRA, 2010). Isso aumenta, ainda mais, os problemas sociais enfrentados por essas comunidades, como o acesso à saúde e ao saneamento básico (FREITAS et al., 2011; CARRIL, 2013; VIEIRA; MONTEIRO, 2013).

A identificação cultural é o primeiro dos desafios, quando se busca realizar pesquisas com comunidades tradicionais. Com os quilombolas isso não é diferente, pois essas comunidades só começaram a receber atenção de estudiosos nas décadas de 1960, 70 e 80. Elas vêm sendo objeto de estudo de cientistas sociais, não só pelo seu percurso sócio histórico, mas pela produção cultural e pela busca cotidiana, no sentido de superar conceitos antiquados que remetem ao passado escravagista (SILVA, 2010).

Do ponto de vista ambiental, os territórios das comunidades tradicionais, em geral, são bem preservados (DIEGUES, 2000), devido à grande relação dessas comunidades com a natureza, onde cada espécie de planta, grupo de animais, tipo de solo e paisagem possuem um uso prático ou religioso (TOLEDO, 1988; FRANCO; BARROS, 2006; MONTELES; PINHEIRO, 2007; CHAGAS et al., 2007; GOMES; BANDEIRA, 2012). Apesar disso, causam impactos em suas terras, principalmente pela caça a animais silvestres (FIGUEIREDO; BARROS, 2016) e pelas ações decorrentes da falta de saneamento básico, como poluição da água (SILVA, 2007).

Diante disso, ações educativas com vistas a reduzir esses impactos têm sido realizadas em diversas comunidades quilombolas (THIAGO, 2011), no intuito de melhorar a qualidade de vida das pessoas que ali habitam (NISHIJIMA; MARTINS, 2010). Assim, se tornam uma ferramenta importante na busca pela identificação cultural e preservação da natureza. Apesar disso, poucos estudos tem sido publicados a respeito dessas ações, dificultando a identificação de lacunas de conhecimento em relação às comunidades quilombolas menos assistidas. Dessa forma, o presente trabalho buscou identificar, quantitativamente, os estudos de educação ambiental realizados em comunidades quilombolas.

\section{Metodologia}

Para a análise quantitativa dos estudos encontrados, foi utilizada a produção bibliográfica como indicadora dos resultados no campo de educação ambiental em comunidades quilombolas dos últimos vinte anos. O levantamento foi realizado na base de 
dados Google Scholar (Google Acadêmico), que foi escolhida por possuir maior número de resultados e fontes altamente citadas, que não são indexadas em outras plataformas, como ISI Web of Science e Scopus (BAR-ILAN, 2007; LEVINE-CLARK; GIL, 2008; KULKARNI et al., 2009; MIKKI, 2010; BERGMAN, 2012; ADRIAANSE; RENSLEIGHT, 2013; ZHU; LIU, 2020). Além disso, é mais intuitiva e indexa uma ampla variedade de documentos que podem ter valor significativo nas análises (MEHO; YANG, 2007).

Em todos os casos, as palavras-chave foram separadas pela palavra and, que retorna resultados que as possam conter ou até mesmo aparecer no mesmo campo, ou em diferentes campos. A busca foi realizada por meio das seguintes palavras-chave: "Quilombolas", "Educação Ambiental" e "Comunidades tradicionais", em português, e no idioma inglês: "Environmental education" e "traditional communities". A palavra "Quilombola" não possui tradução no inglês e, por isso, foi utilizada tanto na busca em português quanto em inglês. A utilização de palavras-chave em mais de um idioma é importante, considerando-se a universalização do inglês no âmbito acadêmico-científico. $\mathrm{O}$ Quadro 1 apresenta resumidamente os critérios de construção da base bibliográfica.

Foi construído um dendrograma por meio de uma análise de agrupamento (Cluster Analysis), utilizando-se o método de média por grupo (Group Average), que considera a média das similaridades dos pares de grupos (nesse caso, tipos de estudos). As análises foram realizadas no software R 3.0.1 (R CORE TEAM, 2013), pacote vegan (OKSANEN et al., 2013). Devido ao modo de exibição do $R$, o gráfico se apresenta como de dissimilaridade, no qual, quanto maior a dissimilaridade, menos similar é o agrupamento.

\begin{tabular}{|c|l|}
\hline \multicolumn{1}{|c|}{ Critério } & \multicolumn{1}{|c|}{ Definição } \\
\hline Base de dados & Google Scholar \\
\hline Tipo de documento & $\begin{array}{l}\text { Artigos, teses, dissertações, notas, resumos em anais } \\
\text { de eventos }\end{array}$ \\
\hline Palavras-chave & $\begin{array}{l}\text { "Quilombola", "Educação Ambiental" e } \\
\text { "Comunidades "tradicionais; "Environmental } \\
\text { education", "quilombolas" e "traditional } \\
\text { communities" }\end{array}$ \\
\hline Áreas de estudo & Sem restrições \\
\hline Período de publicação & Até abril de 2020 \\
\hline Instituição de pesquisa & Sem restrições \\
\hline Língua de publicação & Sem restrições \\
\hline
\end{tabular}

Quadro 1 - Critérios de construção da base bibliográfica

Fonte: adaptada de Cabral-Netto e Laurindo (2015)

Para cada trabalho encontrado nas bases de dados consultadas, foram obtidas as seguintes informações, adaptadas do trabalho de Lima-Ribeiro et al. (2007): (i) ano de publicação; (ii) periódico; (iii) número de citações do artigo; (iv) nacionalidade do primeiro 
autor; (v) área geográfica de enfoque do estudo; (vi) tipo de estudo (teórico, empírico ou descritivo); (vii) comunidade estudada; (viii) tipo do trabalho (resíduos, fauna, flora, saúde, saneamento); (iv) palavras-chave, e (x) tipo de documento publicado (artigos, teses, dissertações, notas, resumos em anais de eventos).

Desse modo, sob esse levantamento proposto, foi possível identificar quais os possíveis significados desse tema abordado, bem como as práticas que estão sendo realizadas no âmbito da educação ambiental dentre as comunidades quilombolas no Brasil. As análises foram estritamente descritivas, por meio de um editor de planilhas (Excel) e dos gráficos elaborados no software Sigma Plot, versão 10.0 (Systat Software Inc.).

\section{Resultado e Discussões}

Foram encontrados, na base de dados consultada, 65 registros contendo as palavras-chaves definidas. A maior parte dos estudos encontrados foi do ano de $2018(\mathrm{n}=11)$. Os anos em que se registrou menor número de estudos encontrados foram 2007 e 2020 (n = 1), com certeza, pelo fato de o presente estudo ter sido realizado ainda no primeiro semestre do ano de 2020 . No entanto, considerando-se as diversas ações e os estudos realizados com comunidades quilombolas, esse número será ainda maior do que no ano de 2018 (Erro! Fonte de referência não encontrada.). $\mathrm{O}$ relativo baixo número de trabalhos encontrados pode ser associado às dificuldades burocráticas que os pesquisadores enfrentam para estudar as comunidades tradicionais no Brasil, o que muitos pesquisadores veem como entrave à pesquisa social (MARRIEL, 2007; TOMANIK, 2013). No entanto, é importante ressaltar que os comitês de ética em pesquisa, regulamentados por meio da Resolução no 196/96 do Conselho Nacional de Saúde, não têm a função de dificultar a pesquisa, mas garantir que ela seja realizada, seguindo protocolos e métodos científicos reconhecidos em estudos com seres humanos. Desse modo, evitam-se conflitos de interesse e respeitam-se as comunidades e os saberes locais (GUERRIERO; MINAYO, 2013; RIBEIRO; FERREIRA, 2016). 


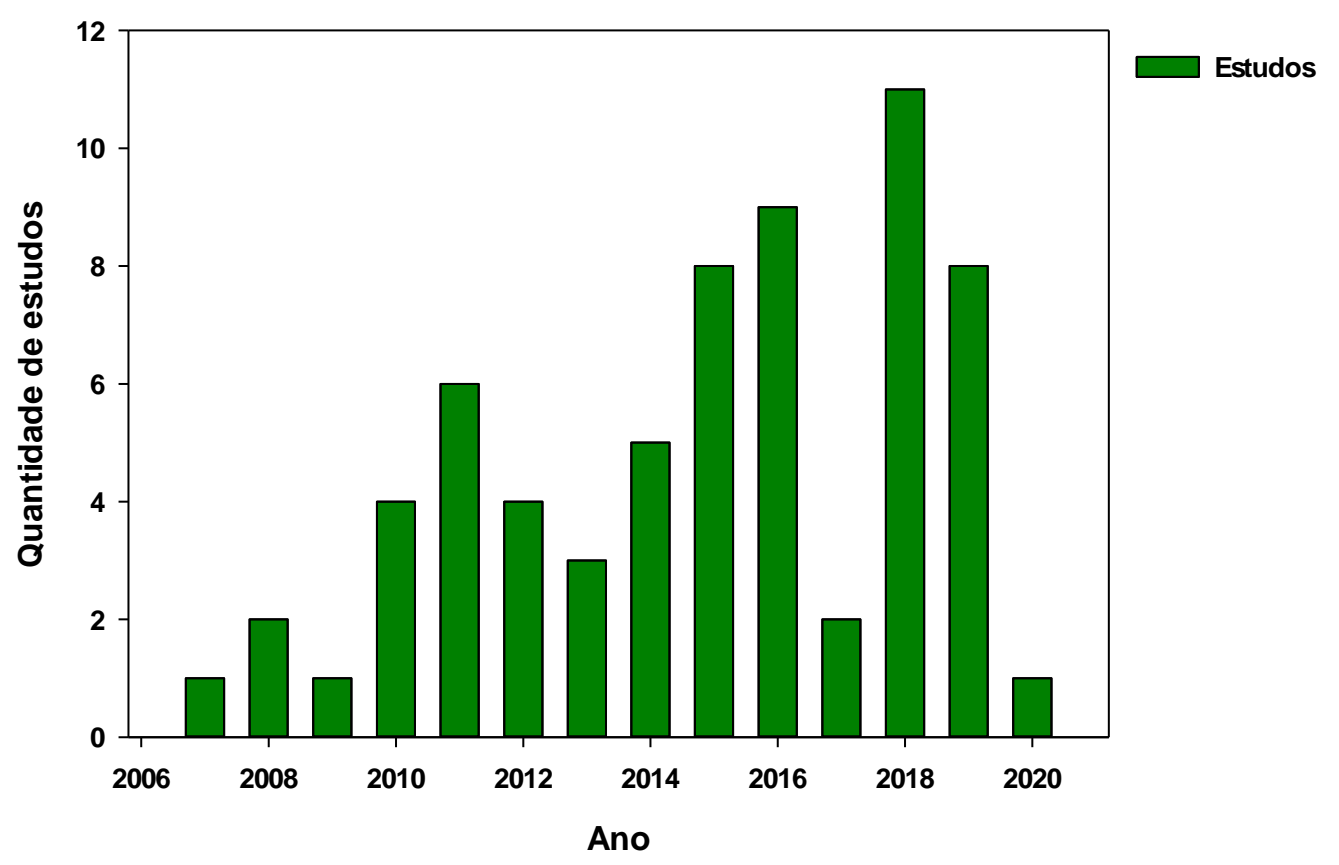

Figura 1 - Quantidade de estudos encontrados. Fonte: Os autores.

De todos os estudos, o tipo mais frequente foi o artigo científico, com 31 registros (47,69\%), seguido do resumo expandido, com treze (20\%), e das dissertações, com onze publicações $(16,92 \%)$. Os tipos relatório e resumo foram os que apresentaram menores valores (1,54\% cada) (Erro! Fonte de referência não encontrada.). A maior parte dos estudos do tipo artigo científico foi publicada nos anos 2015, 2018 e 2019 ( $\mathrm{n}=4$ cada ano), enquanto os resumos foram publicados, com maior frequência, no ano de $2018(n=3)$, possivelmente devido à realização de grandes eventos científicos naquele ano, como o V Congresso Nacional de Educação, onde dois estudos encontrados foram apresentados. 


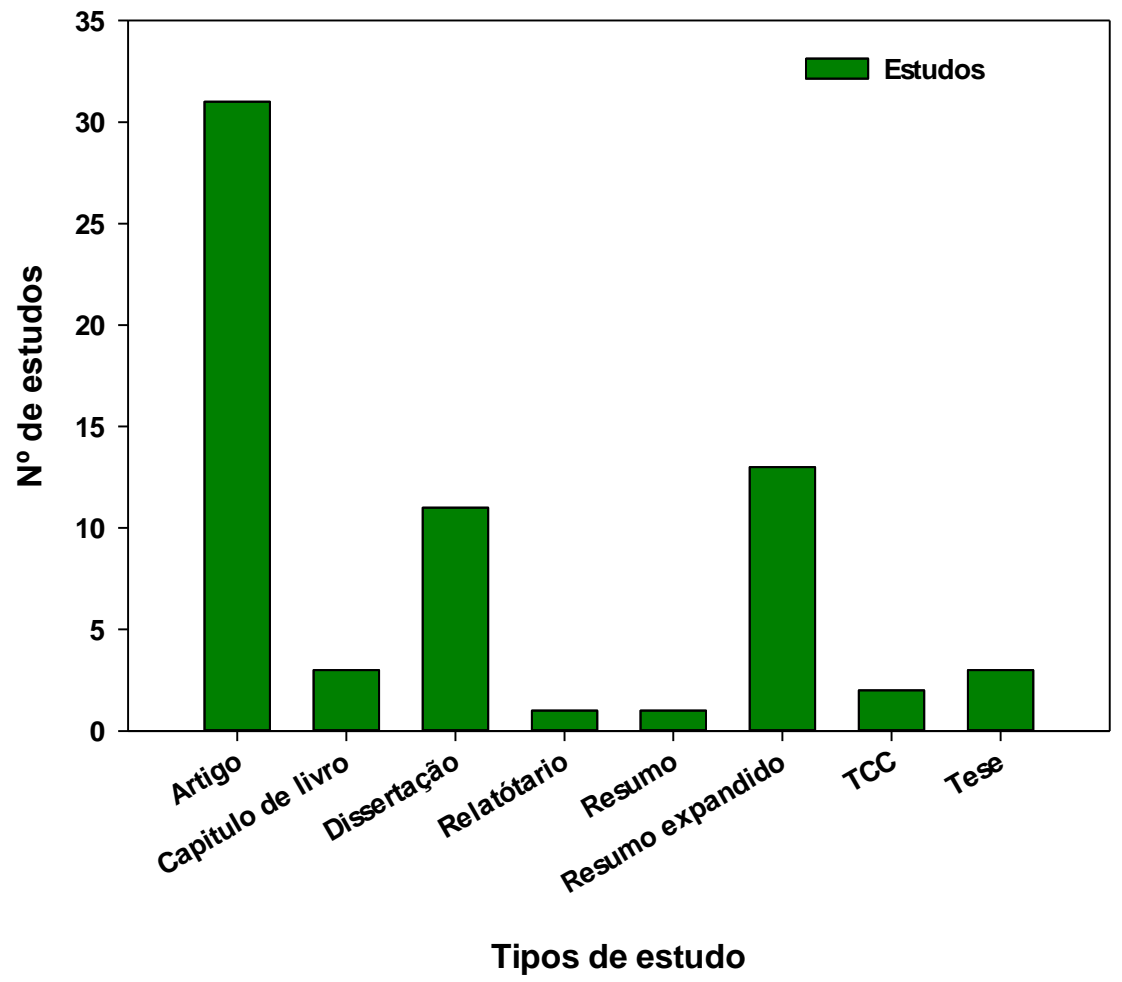

Figura 2 - Tipologia dos estudos encontrados.

Fonte: Os autores.

A partir de uma análise de agrupamentos, foi possível observar que, se considerando os anos de publicação e o tipo de estudo, os tipos resumo expandidos e artigo científico foram $80 \%$ mais similares. Isso significa que, possivelmente, os anos de publicação dos dois tipos foram praticamente os mesmos, enquanto os demais foram bastante dissimilares, indicando que os anos de publicação entre eles foram bastante distintos (Erro! Fonte de referência não encontrada.). Esses dois tipos de estudo também são mais similares quanto à sua estrutura, sendo exigidos, praticamente, os mesmos tópicos nas publicações em anais de eventos. 


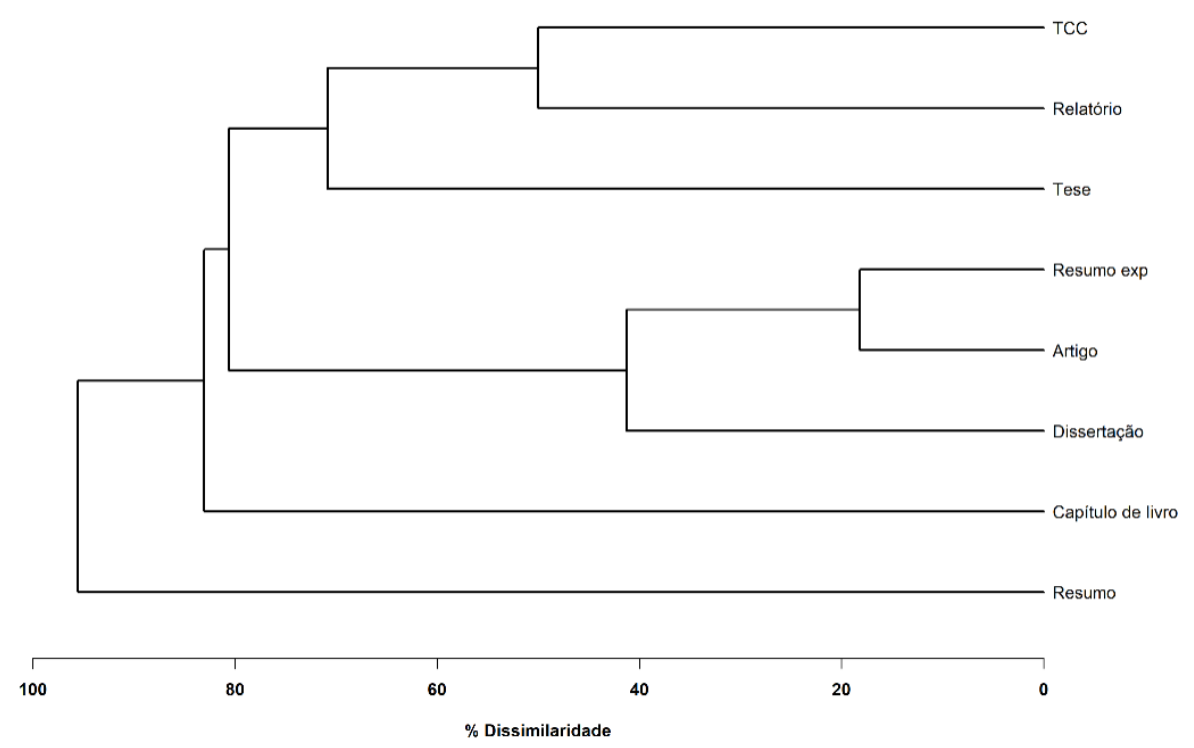

Figura 3 - Dendrograma de dissimilaridade de Jaccard, dos anos dos estudos, com a tipologia destes Fonte: Os autores.

Com relação aos periódicos, o que obteve o maior número de publicações foi a Revista Eletrônica do Mestrado em Educação Ambiental $(n=5)$, que elevou o número de publicações para o Conceito Qualis B2. Apenas um estudo obteve conceito Qualis A2 e outro Qualis C, sendo os dois extremos quanto ao conceito Qualis no Brasil, indicando as revistas de melhor e pior qualidade, respectivamente, por meio desse sistema de avaliação. Outros quatro estudos não possuíam conceito Qualis e, por isso, não foram inseridos nessa análise (Tabela 1). Apesar de criticado por alguns autores (SILVA, 2009; FRIGERI; MONTEIRO, 2014), o conceito Qualis é fundamental para conferir a qualidade dos artigos científicos que são publicados, reconhecendo-se o mérito científico dos periódicos, como dos programas de pós-graduação espalhados pelo Brasil (FRIGERI; MONTEIRO, 2014). Assim, considerando-se a escala definida pela Coordenação de Aperfeiçoamento de Pessoal de Nível Superior (CAPES) durante o Quadriênio 2013-2016, a maioria dos estudos publicados e elencados no presente trabalho pode ser considerada de qualidade intermediária (B2).

Tabela 1 - Periódicos e conceitos Qualis dos estudos publicados

\begin{tabular}{lcc}
\hline \multicolumn{1}{c}{ Periódico } & $\begin{array}{c}\text { Conceito } \\
\text { Qualis }\end{array}$ & $\mathbf{N}^{\mathbf{0}}$ de estudos \\
\hline Agrarian & $\mathrm{B} 2$ & 1 \\
Ambiental Mente Sustentable & $\mathrm{B} 4$ & 1 \\
Antropolítica & $\mathrm{B} 1$ & 1 \\
Espacios & $\mathrm{C}$ & 1 \\
EXTRAMUROS & $\mathrm{B} 4$ & 1 \\
Interfaces Científicas & $\mathrm{B} 4$ & 1 \\
Investigações em Ensino de Ciências & $\mathrm{A} 2$ & 1 \\
Olhar de Professor & $\mathrm{B} 3$ & 1 \\
\hline
\end{tabular}




\begin{tabular}{lll}
\hline Percursos & B4 & 1 \\
REDES - Revista do Desenvolvimento Regional & B2 & 2 \\
Revista Acervo & B4 & 1 \\
Revista Brasileira de Ciências Ambientais (Online) & B1 & 1 \\
Revista Brasileira de Ecoturismo & B3 & 1 \\
Revista de Educação Pública & B3 & 1 \\
Revista Educação Ambiental em Ação & B3 & 1 \\
Revista Eletrônica Associação dos Geógrafos Brasileiros & B5 & 1 \\
Revista Eletrônica do Mestrado em Educação Ambiental & B2 & 5 \\
Revista Eletrônica em gestão, Educação Tecnologia Ambiental & B2 & 1 \\
Revista Eletrônica TEMPUS & B4 & 1 \\
Revista Monografias Ambientais & B4 & 1 \\
Revista Odeere & B5 & 1 \\
UNITAS - Revista Eletrônica de Teologia e Ciências das & B5 & 1 \\
Religiões & \\
\hline
\end{tabular}
Fonte: Os autores.

Já os temas dos trabalhos publicados foram extremamente variados, de Antropologia até produção de cerâmica e teatro. Percebe-se, assim, que a educação ambiental está inserida em vários contextos, demonstrando não ter uma funcionalidade única e, muito menos, uma definição única (Quadro 2). De fato, a educação ambiental, em si, é multidisciplinar e abrange várias áreas do conhecimento (GUIMARÃES, 1995; JACOBI, 2005; GAVIÃO; LIMA, 2014). Como é possível ver no Quadro 2, os temas mais publicados se relacionam à ecologia e à flora ( $\mathrm{n}=3$ cada).

Outro fator a ser considerado, e que pode ter contribuído para a grande diversidade de temas abordados, refere-se à característica histórica de formação dos quilombos. Segundo Silva e Souza (2021), os quilombos mantêm vínculos profundos de sua identidade com os seus territórios tradicionais, seja pelos modos de vida, seja pelos processos de resistência à opressão histórica sofrida. Essa identidade e os modos de vida refletem, também, a diversidade de possibilidades de temas para estudo pelos pesquisadores.

\begin{tabular}{|l|l|}
\hline \multicolumn{1}{|c|}{ Tema do estudo } & \multicolumn{1}{c|}{ Título do estudo } \\
\hline Ações de educação ambiental & $\begin{array}{l}\text { Educação ambiental: um olhar sobre comunidades quilombolas na } \\
\text { região central do Rio Grande do Sul. }\end{array}$ \\
\hline Antropologia & $\begin{array}{l}\text { Bases antropológicas para projetos de educação ambiental no } \\
\text { quilombo do Cabral, em Paraty, RJ. }\end{array}$ \\
\hline Condicionante ambiental & $\begin{array}{l}\text { A educação ambiental crítica e o projeto de mitigação em } \\
\text { comunidades quilombolas: o caso do projeto Quipea. }\end{array}$ \\
\hline Conservação ambiental & $\begin{array}{l}\text { Análise do estado de conservação ambiental na comunidade } \\
\text { quilombola da Resina, Brejo Grande/SE: a influência do rio São } \\
\text { Francisco na vida dos ribeirinhos. }\end{array}$ \\
\hline Desenvolvimento sustentável & $\begin{array}{l}\text { Indicadores de sustentabilidade na comunidade quilombola } \\
\text { África, município de Abaetetuba, Pará, Brasil. }\end{array}$ \\
\hline EA e alterações climáticas & $\begin{array}{l}\text { Água e vento são meio de sustento: aspectos teórico-conceituais a } \\
\text { serem considerados na pesquisa em Educação Ambiental e } \\
\text { mudanças climáticas. }\end{array}$ \\
\hline Ecologia & $\begin{array}{l}\text { Comunidade quilombola Barra da Aroeira (TO): abordagem } \\
\text { fenomenológica das práticas ecológicas. }\end{array}$ \\
\hline
\end{tabular}




\begin{tabular}{|c|c|}
\hline & $\begin{array}{l}\text { Ecologia de saberes: a experiência do diálogo entre conhecimento } \\
\text { científico e conhecimento tradicional na comunidade quilombola } \\
\text { da Rocinha. }\end{array}$ \\
\hline Ecoturismo & $\begin{array}{l}\text { Ecoturismo de Base Comunitária na Comunidade Quilombola } \\
\text { Furnas da Boa Sorte, Corguinho (MS): planejamento e } \\
\text { sustentabilidade. }\end{array}$ \\
\hline Educação do campo & $\begin{array}{l}\text { Educação do campo, desenvolvimento sustentável na comunidade } \\
\text { quilombola do Serrote. }\end{array}$ \\
\hline Educação moral & A moral ambiental em crianças quilombolas. \\
\hline Ensino de ciências & $\begin{array}{l}\text { Conhecimentos tradicionais e o ensino de ciências na educação } \\
\text { escolar quilombola: um estudo etnobiológico. }\end{array}$ \\
\hline Estudos de impactos ambientais & $\begin{array}{l}\text { Estudo de impactos ambientais na comunidade quilombola Serra } \\
\text { Feia-Cacimbas, PB. }\end{array}$ \\
\hline Etnografia e Etnobotânica & $\begin{array}{l}\text { Tecendo histórias etnográficas e etnobotânicas na comunidade } \\
\text { quilombola do Cedro em Mineiros, Goiás. }\end{array}$ \\
\hline \multirow{3}{*}{ Flora } & $\begin{array}{l}\begin{array}{l}\text { Conhecimento quilombola e plantas medicinais: recursos } \\
\text { didáticos para o ensino de ciências. }\end{array} \\
\end{array}$ \\
\hline & $\begin{array}{l}\text { Educação ambiental: diálogo de saberes populares sobre ervas } \\
\text { medicinais na comunidade quilombola Areal da Baronesa-Porto } \\
\text { Alegre, RS. }\end{array}$ \\
\hline & $\begin{array}{l}\text { Plantas medicinais e conhecimento tradicional quilombola: um } \\
\text { diálogo com a educação ambiental. }\end{array}$ \\
\hline Gênero & Cultura e arte da mulher negra no contexto da educação ambiental. \\
\hline \multirow[t]{2}{*}{ Identidade cultural } & $\begin{array}{l}\text { A caminhada das mulheres quilombolas de mata cavalo } \\
\text { delineando seu território por entre as trilhas da educação } \\
\text { ambiental. }\end{array}$ \\
\hline & O reconhecimento do povo quilombola de Cachoeira Porteira. \\
\hline Mapeamento participativo & $\begin{array}{l}\text { O mapa social e a educação ambiental, diálogos de um } \\
\text { mapeamento participativo no Pantanal, Mato Grosso, Brasil. }\end{array}$ \\
\hline Participação comunitária & $\begin{array}{l}\text { Ligando os pontos: petróleo, educação ambiental e mobilização } \\
\text { quilombola na Bacia de Campos, Brasil. }\end{array}$ \\
\hline Práticas culturais & $\begin{array}{l}\text { Latas d'água nas cabeças: percepções sobre a água na comunidade } \\
\text { quilombola de Mata Cavalo. }\end{array}$ \\
\hline Preservação Ambiental & $\begin{array}{l}\text { Preservação ambiental e qualidade de vida em comunidades } \\
\text { quilombolas. }\end{array}$ \\
\hline Produção de cerâmica & $\begin{array}{l}\text { Estratégias sustentáveis de produção tradicional de cerâmica na } \\
\text { comunidade quilombola de Itamatatiua, Alcântara/MA. }\end{array}$ \\
\hline Reconhecimento do povo & Racismo Ambiental na Comunidade Quilombola de Mata Cavalo. \\
\hline Revitalização ambiental & $\begin{array}{l}\text { O projeto de revitalização ambiental do córrego angico e a } \\
\text { educação para sustentabilidade na comunidade quilombola } \\
\text { Malhadinha-Tocantins. }\end{array}$ \\
\hline Saneamento & $\begin{array}{l}\text { Saneamento como ferramenta para a sustentabilidade da área } \\
\text { quilombola Vila Esperança, Lapa, PR. }\end{array}$ \\
\hline Solos & $\begin{array}{l}\text { Inserindo o solo na educação ambiental: uma experiência no } \\
\text { Centro-Oeste. }\end{array}$ \\
\hline Teatro & $\begin{array}{l}\text { O teatro como forma de atuação da educação ambiental para a } \\
\text { emancipação política no quilombo de Mata Cavalo. }\end{array}$ \\
\hline
\end{tabular}

Fonte: Os autores.

Do total de estudos (65), 58 informaram quais comunidades quilombolas foram estudadas (Tabela 2).

Tabela 2 - Comunidades onde os estudos encontrados foram realizados

\begin{tabular}{rcc}
\hline Comunidade & Estado & Estudos encontrados \\
\hline Comunidade de Cachoeira Porteira & PA & 1 \\
\hline
\end{tabular}




\begin{tabular}{|c|c|c|}
\hline Comunidade do Quilombo Maria Conga & RJ & 1 \\
\hline Comunidade Kalunga & $\mathrm{GO}$ & 2 \\
\hline Comunidade Quilombo Paraty & RJ & 1 \\
\hline Comunidade Quilombola África & PA & 1 \\
\hline Comunidade Quilombola Caiana dos Crioulos & PB & 1 \\
\hline Comunidade Quilombola Cruz da Menina & PB & 1 \\
\hline Comunidade Quilombola da Barra da Aroeira & TO & 1 \\
\hline Comunidade Quilombola da Picadinha-Itahum & MS & 1 \\
\hline Comunidade Quilombola da Rocinha & BA & 1 \\
\hline Comunidade Quilombola da Serra do Evaristo & $\mathrm{CE}$ & 2 \\
\hline Comunidade Quilombola de Barreiros & BA & 1 \\
\hline Comunidade Quilombola de Castainho & $\mathrm{PE}$ & 1 \\
\hline Comunidade Quilombola de Ernesto Penna Carneiro & RS & 1 \\
\hline Comunidade Quilombola de Itamatatiua & MA & 1 \\
\hline Comunidade Quilombola de Malgada Grande & MG & 1 \\
\hline Comunidade Quilombola de Mata Cavalo & MT & 2 \\
\hline Comunidade Quilombola de Monte Alegre & ES & 2 \\
\hline Comunidade Quilombola de Resina & SE & 1 \\
\hline Comunidade Quilombola de São Pedro de Cima & MG & 2 \\
\hline Comunidade Quilombola do Cedro & $\mathrm{GO}$ & 2 \\
\hline Comunidade Quilombola do Mumbuca & TO & 1 \\
\hline Comunidade Quilombola do serrote & $\mathrm{PE}$ & 1 \\
\hline Comunidade Quilombola Dom João & BA & 1 \\
\hline Comunidade Quilombola Engenho Siqueira & $\mathrm{PE}$ & 1 \\
\hline Comunidade Quilombola Furnas da Boa Sorte & MS & 1 \\
\hline Comunidade Quilombola Malhadinha & TO & 2 \\
\hline Comunidade Quilombola Manoel Barbosa & RS & 1 \\
\hline Comunidade Quilombola Mata-Cavalo & MT & 8 \\
\hline Comunidade Quilombola Mesquita & $\mathrm{GO}$ & 1 \\
\hline Comunidade Quilombola Murumuru & PA & 1 \\
\hline Comunidade Quilombola Muvuca & SE & 1 \\
\hline Comunidade Quilombola Pai André & MT & 1 \\
\hline Comunidade Quilombola Paiol da Telha & PR & 1 \\
\hline Comunidade Quilombola Passo do Maia & RS & 1 \\
\hline Comunidade Quilombola Patioba 1 e 2 & SE & 1 \\
\hline Comunidade quilombola Serra feia & PB & 1 \\
\hline Comunidade Quilombola Vila do Forte & $\mathrm{GO}$ & 1 \\
\hline Comunidade Quilombola Vila esperança & PR & 1 \\
\hline Conceição das Crioulas & $\mathrm{PE}$ & 1 \\
\hline Furnas do Dionísio & MS & 1 \\
\hline Quilombo Areal da Baronesa & RS & 1 \\
\hline Quilombo da Barra da Aroeira & TO & 1 \\
\hline RESEX do Quilombo Frechal & MA & 1 \\
\hline
\end{tabular}

Fonte: Os autores.

Do ponto de vista espacial, as comunidades estudadas abrangem todo o território nacional, sendo que a maior quantidade de estudos foi realizada no estado do Mato Grosso/MT, na comunidade Quilombola Mata-Cavalo ( $\mathrm{n}=8$, Tabela 2). A Comunidade Quilombola MataCavalo está localizada em Nossa Senhora do Livramento/MT e conta, atualmente, com aproximadamente 418 famílias, que se reconhecem como descendentes de pessoas escravizadas e que vivem em uma área de cerca de 14.690,3413 hectares (PALMARES, 2020).

O reflexo dos estudos realizados na Comunidade Quilombola Mata-Cavalo também é visto quando se observa a instituição dos autores. O maior número de estudos foi comandado por pesquisadores da Universidade Federal do Mato Grosso ( $n=9)$, representando $14,52 \%$ dos 
estudos publicados, sendo, em seguida, os estudos comandados por pesquisadores da Universidade de Brasília ( $\mathrm{n}=6$; 9,68\%), Universidade Federal do Rio Grande do Sul e Universidade Federal do Tocantins ( $\mathrm{n}=3 ; 4,84 \%$ cada) (Erro! Fonte de referência não encontrada.).

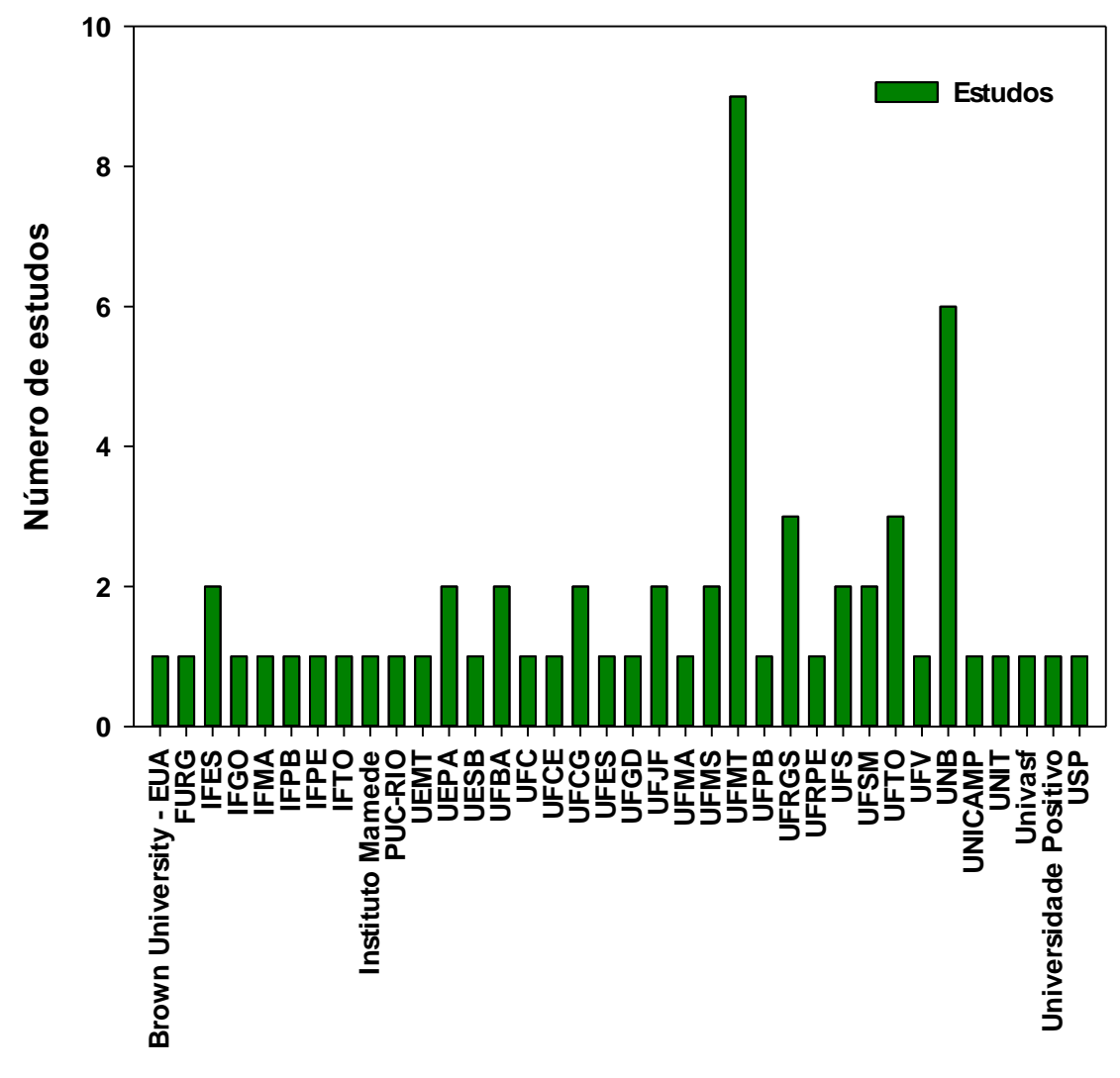

Instituições

Figura 2 - Instituições do primeiro do autor Fonte: Os autores.

Em tempos de desvalorização da ciência e das universidades federais, cabe ressaltar a importância dessas instituições com relação a estudos sociais, sendo que apenas um estudo, do total de 65, foi realizado por uma universidade particular (Universidade Positivo). Por isso, para que esses estudos não cessem e essas comunidades fiquem desamparadas, é necessário investir na educação pública, bem como na pós-graduação, que representou quase a totalidade dos estudos $(n=60)$.

\section{Conclusões}

As comunidades quilombolas sofrem, há anos, com desapropriações e a falta de reconhecimento. Os estudos encontrados mostram que, apesar de poucas instituições no Brasil 
se dedicarem a esse grupo como pesquisa, estas não são desassistidas do ponto de vista acadêmico. Foram registrados 65 estudos envolvendo educação ambiental com diversas comunidades quilombolas espalhados pelo país, com destaque para o maior número de estudos registrados na Comunidade Quilombola Mata-Cavalo, no estado do Mato Grosso, uma comunidade que, desde sua criação, vem sofrendo com constantes desapropriações.

A maior parte dos estudos encontrados foi do tipo artigo científico, em grande parte, no ano de 2018. Apesar de poucos estudos terem sido publicados, a qualidade destes pode ser considerada intermediária, considerando-se os critérios da CAPES. O maior número de publicações foi encontrado na Revista Eletrônica do Mestrado em Educação Ambiental, vinculada ao Programa de Pós-Graduação em Educação Ambiental da FURG.

Os temas dos estudos encontrados foram bastante variados, demonstrando o caráter multidisciplinar da educação ambiental. Associadas a isso, há características históricas de formação dos quilombos, cujos habitantes mantêm vínculos profundos de sua identidade com os seus territórios tradicionais, seja pelos modos de vida, seja pelos processos de resistência à opressão histórica sofrida. Essa identidade e os modos de vida refletem, também, a diversidade de possibilidades de temas para estudo pelos pesquisadores.

Dito isso, e considerando-se o fato de os estudos terem sido realizados, quase que em sua totalidade, por instituições públicas de ensino, é necessário defender essas instituições por serem extremamente necessárias para as comunidades quilombolas, ao incentivarem a realização de diversos estudos das mais diversas naturezas.

\section{Referências}

ADRIAANSE, L.; RENSLEIGH, C. Web of Science, Scopus and Google Scholar. The Electronic Library, v. 31, n. 6, p. 727-744, 2013.

ALMEIDA, Silvio Luiz de. O que é racismo estrutural? Belo Horizonte: Letramento, 2018. Não aparece no texto - suprimir

AMORIM, I. G.; GERMANI, G. I. Quilombos da Bahia: presença incontestável. In: ENCONTRO DE GEÓGRAFOS DA AMÉRICA LATINA, 10, 2005, São Paulo. Anais... São Paulo: Universidade de São Paulo, 2005. p. 796-812. Disponível em:

https://geografar.ufba.br/sites/geografar.ufba.br/files/geografar_amorimgermani_quilombosbahia.pdf

BAR-ILAN, J. Which h-index? - A comparison of WoS, Scopus and Google Scholar. Scientometrics, [s.1], v. 74, n. 2, p. 257-271, 2008.

BERGMAN, L. E. M. Finding Citations to Social Work Literature: The Relative Benefits of Using Web of Science, Scopus, or Google Scholar. The Journal of Academic Librarian ship, [s.1], v. 38, n. 6, p. 370-379, 2012. 
CABRAL, E. R.; DOS SANTOS, A. L. L.; GOMES, S. C. Responsabilidade Social e Ambiental e Desenvolvimento local Sustentável: O Caso do Projeto de Educação Ambiental e Patrimonial-PEAP. Revista de Gestão Ambiental e Sustentabilidade, São Paulo, v.4, n. 1, p. 91-107, 2015.

CARRIL, L. Quilombo, favela e periferia: a longa busca da cidadania. São Paulo: Annablume/ FAPESP, 2013.

CHAGAS, M. C. C.; DE ANDRADE, M. G.; DA COSTA, R. B.; DE SOUZA PERRELLI, M. A. A prática de benzimento com uso de plantas na comunidade rural remanescente de quilombo de Furnas do Dionísio, Jaraguari, Mato Grosso do Sul. Multitemas, Campo Grande, Mato Grosso do Sul, n. 35, p. 207-224, 2007.

DIEGUES, A. C. A etnoconservação da natureza. In: DIEGUES, A. C. (Org.). Etnoconservação: novos rumos para a proteção da natureza nos trópicos. 2. ed. São Paulo: Hucitec/NUPAUB, 2000. p. $1-46$.

FIGUEIREDO, R. A. A. D.; BARROS, F. B. Caçar, preparar e comer o ‘bicho do mato': práticas alimentares entre os quilombolas na Reserva Extrativista Ipaú-Anilzinho (Pará). Boletim do Museu Paraense Emílio Goeldi, Belém, Pará, v.11, n. 3, p. 691-713, 2016.

FRANCO, E. A. P.; BARROS, R. F. M. Uso e diversidade de plantas medicinais no Quilombo Olho D’água dos Pires, Esperantina, Piauí. Revista Brasileira de Plantas Medicinais, Botucatu, v. 8, n. 3, p. 78-88, 2006.

FREITAS, D. A.; SILVEIRA, J. C. S.; FERREIRA, L. A.; ZUCCHI, P.; MARQUES, A. S. Mulheres Quilombolas: profissionais na estratégia de saúde da família. Espaço Para Saúde, Curitiba, v. 12, n. 2, p. 56-62, 2011.

FRIGERI, M.; MONTEIRO, M. S. A. Qualis Periódicos: indicador da política científica no Brasil? Estudos de Sociologia, Recife, v.19, n. 37, p. 299-315, 2014.

FUNDAÇÃO PALMARES. Comunidades Quilombolas do Brasil. 2020. Brasília: Fundação palmares, 2020. Disponível em: http://www.palmares.gov.br/?page_id=37551. Acesso em: 29 abr. 2020.

GAVIÃO, L. O.; LIMA, G. B. A. Diagnóstico Multidisciplinar da Educação Ambiental no Ensino Médio Brasileiro: aplicação de indicadores de desempenho em uma escola de Niterói (RJ). Ensino, Saúde e Ambiente, Niterói, v.7, n. 2, p. 46-63, 2014.

GOMES, T. B.; BANDEIRA, F. P. S. D. F. Uso e diversidade de plantas medicinais em uma comunidade quilombola no Raso da Catarina, Bahia. Acta Botanica Brasilica, São Paulo, v. 26, n. 4, p. $796-809,2012$.

GUERRIERO, I. C. Z.; MINAYO, M. C. D. S. O desafio de revisar aspectos éticos das pesquisas em ciências sociais e humanas: a necessidade de diretrizes específicas. Physis: Revista de Saúde Coletiva, Rio de janeiro, v. 23, n. 3, p. 763-782, 2013.

GUIMARÃES, M. A dimensão ambiental na educação. São Paulo: Papirus, 1995.

JACOBI, P. R. Educação ambiental: o desafio da construção de um pensamento crítico, complexo e reflexivo. Educação e pesquisa, São Paulo, v. 31, n. 2, 233-250, 2005.

KULKARNI, A. V.; AZIZ, B.; SHAMS, I.; BUSSE, J. W. Comparisons of citations in Web of Science, Scopus, and Google Scholar for articles published in general medical journals. Jama, [s.1], v. 302, n.10, p.1092-1096, 2009. 
LEITE, I. B. Humanidades insurgentes: conflitos e criminalização dos quilombos. In: ALMEIDA, Alfredo Wagner et al. (Orgs.). Cadernos de debates Nova Cartografia Social: territórios quilombolas e conflitos. Manaus: Projeto Nova Cartografia Social da Amazônia/UEA Edições, 2010. p. 18-40.

LEVINE-CLARK, M.; GIL, E. L. A Comparative Citation Analysis of Web of Science, Scopus, and Google Scholar. Journal of Business \& Finance Librarian ship, [s.1], v.14, n.1, p. 32-46, 2008.

SOUZA LIMA-RIBEIRO, M., NABOUT, J. C., PINTO, M. P., DE MELO, T. L., COSTA, S. S., \& DE BRITTO, T. F. L. V. Análise cienciométrica em ecologia de populações: importância e tendências dos últimos 60 anos. Acta Scientiarum. Universidade Estadual de Maringá. Biological Sciences, v. 29 n. 1, 39-47, 2007.

MARRIEL, N. Comitês de ética em pesquisa: burocracia ou parceria? Uma visão pedagógica do papel educativo dos comitês no processo das pesquisas. Revista de educação do Cogeime, São Paulo, v.16, n. 31, p. 21-36, 2007.

MEHO, L. I.; YANG, K. Impact of data sources on citation counts and rankings of LIS faculty: Web of science versus scopus and google scholar. Journal of the American Society for Information Science and Technology, [s.1], v. 58, n. 13, p. 2105-2125, 2007.

MIKKI, S. Comparing Google Scholar and ISI Web of Science for Earth Sciences. Scientometrics, [s.1], v. 82, n. 2, p. 321-331, 2010.

MONTELES, R.; PINHEIRO, C. U. B. Plantas medicinais em um quilombo maranhense: uma perspectiva etnobotânica. Revista de Biologia e Ciências da Terra, v.7, n. 2, p. 38-48, 2007.

NISHIJIMA, T.; MARTINS, L. A. R. Preservação ambiental e qualidade de vida em comunidades Quilombolas. Revista Eletrônica em Gestão, Educação e Tecnologia Ambiental, João Pessoa, v.1, n.1, p. 59-69, 2010.

OKSANEN, J. et al. Vegan: Community Ecology Package. R package version 2.0-7. 2013. Disponível em: http://CRAN.R-project.org/packag e=vegan.

OLIVEIRA, O. M. de. Quilombos: memória social e metáforas dos conflitos comunidades do Sapê do Norte, Espírito Santo. In: ALMEIDA, Alfredo Wagner et al. (Orgs.). Cadernos de debates Nova Cartografia Social: Territórios quilombolas e conflitos. Manaus: Projeto Nova Cartografia Social da Amazônia / UEA Edições, 2010. p. 63-69.

R CORE TEAM. R: A language and environment for statistical computing. Vienna: R Foundation for Statistical Computing, 2013. Disponível em: http://www.R-project.org/. Acesso em: 05 de maio de 2020.

RIBEIRO, S. A. B.; FERREIRA, S. B. L. Pesquisa Envolvendo Seres Humanos: Comitê De Ética e Pesquisa. Guia. UNIRIO - Universidade Federal do Estado do Rio de Janeiro -Centro de Ciências Exatas e Tecnologia. p. 1-8. (2016). Disponível em:

http://nau.uniriotec.br/images/pdf/guia/tutorialComiteEtica.pdf. Acesso em: 6 abr. 2020.

SILVA, A. O. A sua revista tem Qualis? Mediações-Revista de Ciências Sociais, Londrina, v.14, n.1, p. 117-124, 2009.

SILVA, R. L. F. Leitura de imagens da mídia e educação ambiental: contribuições para a formação de professores. Educação em Revista, v. 26, n. 2. 277-297, 2010.

SILVA, J. A. N. D. Condições sanitárias e de saúde em Caiana dos Crioulos, uma comunidade Quilombola do Estado da Paraíba. Saúde e Sociedade, Ribeirão Preto, v. 16, n. 2, p. 111-124, 2007. 
SILVA, G. M. DA; SOUZA, B. O. Quilombos e a Luta contra o Racismo no Contexto da Pandemia. Boletim de Análise Político-Institucional. Repositório do IPEA, [s.v], n. 26, p. 85-91, 2021.

SOUZA, T. G. D., \& LARA, L. M. O estado da arte de comunidades quilombolas no Paraná: produção de conhecimento e práticas corporais recorrentes. Revista da Educação Física/UEM, v. 22, n. 2, 555568, 2011.

THIAGO, F. A comunidade quilombola do Cedro, Mineiros-GO: etnobotânica e educação ambiental. 2011. 109 f. Dissertação (Mestrado em Ciências Ambientais) - Universidade do Estado de Mato Grosso, Cáceres, 2011.

TOLEDO, V. M. La diversidad biológica de México. Ciencia y Desarrollo, Lima, v. 81, [s.n], p. 1730, 1988.

TOMANIK, E. A. Debatendo os comitês de ética: controle burocrático ou laissez-faire? Psicologia em Estudo, Natal, v.18, n.1, p. 175-180, 2013.

VIEIRA, A. B. D.; MONTEIRO, P. S. Comunidade quilombola: análise do problema persistente do acesso à saúde, sob o enfoque da Bioética de Intervenção. Saúde em Debate, Rio de Janeiro, v. 37, [s.n], p. 610-618, 2013.

ZHU, J.; LIU, W. A tale of two data bases: the use of Web of Science and Scopus in academic papers. Scientometrics, [s.1], v.123, [s.n]. p. 321-335, 2020. Disponível em: https://arxiv.org/abs/2002.02608 